\title{
Motivação Acadêmica e Autoestima Contingente: Relação com Satisfação de Vida, Esperança e Otimismo
}

\author{
Daniela Bergesch D'Incao Marrone ${ }^{1}$, Claudio Simon Hutz \\ Universidade Federal do Rio Grande do Sul, Porto Alegre-RS, Brasil
}

\section{RESUMO}

Este estudo investigou em uma amostra de estudantes de universidades públicas e privadas a relação entre o tipo de motivação para os estudos (intrínseca ou externa) com autoestima e personalidade, otimismo, esperança e satisfação de vida. Os resultados indicaram associação entre os domínios de competência acadêmica, competição e aparência com Motivação Extrínseca no âmbito acadêmico. Além disso, houve associação significativa do fator neuroticismo com o domínio Aprovação de Outros. As mulheres apresentaram escores mais elevados em domínios de contingência externa. Foram realizadas análises de regressão e o domínio Aprovação de Outros demonstrou maior poder de explicação (35,9\%), seguido pelo de Competência Acadêmica (28,4\%) e Aparência (25,5\%). Esses achados estão de acordo com a literatura, indicando que quando a autoestima se apoia em contingências externas pode haver menor motivação para os estudos. Os achados indicam a necessidade de estratégias de intervenção que propiciem ambientes que oportunizem o interesse intrínseco para a aprendizagem nas universidades.

Palavras-chave: motivação acadêmica; autoestima contingente; satisfação de vida; esperança; otimismo.

\section{ABSTRACT - Academic Motivation and Contingent Self-Esteem: Investigating the Relation with Life Satisfaction, Hope and Optimism}

Motivation for learning is fundamental and closely related to external and internal factors and to the extent to which they interrelate. Accordingly, self-esteem can be an indicator of how the individual deals with the environment and with internal pressure, especially when this is based on external contingencies. This study sought to investigate, in a sample of university students, the relationship of the type of motivation with self-esteem and characteristics such as personality, optimism, hope, and life satisfaction. In the study sample, an association was found between extrinsic motivation in the academic environment and the contingency domains of academic competence, competition, and appearance. We concluded that the findings are in line with the literature and indicate the need for intervention strategies in higher education, aiming to promote an environment that is more favorable to the intrinsic interest in studying.

Keywords: academic motivation; contingent self-esteem; life satisfaction; hope; optimism.

\section{RESUMEN - Motivacón Académica y Autoestima Contingente: Relación con la Satisfaccíon de La Vida, La Esperanza y el Optimismo}

La motivación para el aprendizaje es fundamental y está estrechamente relacionada con factores extrínsecos e intrínsecos y cómo estos se interrelacionan. En este sentido, la autoestima puede ser un indicador de cómo el individuo trata con el medio ambiente y con las presiones internas, especialmente cuando depende de contingencias externas como una forma de mantener el autoestima elevado. Este estudio tuvo como objetivo investigar en una muestra de estudiantes universitarios la relación del tipo de motivación para los estudios (intrínseca o extrínseca) con autoestima y características tales como personalidad, optimismo, esperanza y satisfacción con la vida. La muestra del estudio presentó una asociación entre los dominios de competencia académica, competición y apariencia con la motivación extrínseca en el campo académico. Se concluye que los resultados respaldan la literatura vigente, lo que indica la necesidad de estrategias de intervención en el ámbito de la educación superior para promover un ambiente más propicio para el interés intrínseco para el aprendizaje en las universidades.

Palabras clave: motivación académica; autoestima contingente; satisfacción con la vida; esperanza; optimismo.

Durante o processo de aprendizagem, a motivação é fundamental e tem demonstrado capacidade de predizer variância de satisfação em nível educacional em diferentes áreas (Gilman \& Anderman, 2006; Lin, Mckeachie,
\& Kim, 2001; McDermott, Mordell, \& Stoltzfus, 2001). A motivação foi, durante muito tempo, compreendida em termos de contingências extrínsecas, ou seja, existiria um reforçador arbitrário que antecederia um 
tipo de comportamento (Lepper \& Henderlong, 2000). Entretanto, há evidências de que quando são oferecidas recompensas a um comportamento, isso pode gerar o oposto do desejado: a diminuição da motivação intrínseca (Lepper \& Henderlong, 2000).

As dificuldades encontradas no contexto universitário, dentre elas as de aprendizagem, dizem respeito a questões pessoais que podem interferir na motivação para aprender (Cunha \& Carrilho, 2005). Uma das formas de identificar o tipo de motivação presente no processo de aprendizagem é a autoestima. Quanto mais a autoestima de um estudante se apoiar no sucesso acadêmico, mais ela aumentará quando este obtiver boas notas (Crocker, Karpinski, Quinn, \& Chase, 2003), bem como haverá maior vulnerabilidade quando não houver obtenção dos resultados almejados.

Cabe ressaltar que o que pode ser de extrema importância para uma pessoa pode não ter relevância alguma na vida de outra, vai depender da autoavaliação que cada um faz acerca das áreas julgadas relevantes (Maricutoiu, Macsinga, Magurean, Virga, \& Sava, 2012). As contingências de autoestima representam domínios nos quais a autoestima é ameaçada por falhas e/ou reveses e, nestas circunstâncias, as pessoas estão mais propensas a apresentar condutas defensivas (Crocker \& Wolfe, 2001). Nesse sentido, quanto mais contingente, mais frágil é a autoestima (Crocker \& Park, 2004; Sanchez \& Crocker, 2005) com risco aumentado para problemas como ansiedade e depressão (Crocker, Karpinski, Quinn, \& Chase, 2003; Lopez et al., 2014).

Cada comportamento, portanto, é motivado por variações potenciais nos seus níveis usuais de autoestima, não podendo esta ser considerada somente como baixa ou alta (Crocker \& Wolfe, 2001). A motivação para que o indivíduo faça suas escolhas pode se dar, em termos gerais, de duas formas: externamente ou intrinsecamente. Quando a motivação se dá por motivos externos, há uma busca por atender certas demandas (Ryan \& Deci, 2000). Por outro lado, quando a motivação é intrínseca (autêntica), ela geralmente engloba sentimentos de interesse, empolgação e confiança (Ryan \& Deci, 2000), podendo acarretar o incremento de criatividade (Sheldon, Ryan, Rawsthorne, \& Ilardi, 1997), bem-estar (Ryan, Deci, \& Grolnick, 1995) e autoestima (Deci \& Ryan, 1995).

A motivação intrínseca é facilitadora da aprendizagem. A literatura aponta que ambientes que propiciam suporte a comportamentos autônomos, como, por exemplo, pais e professores que dão suporte à autonomia dos indivíduos, facilitam a motivação intrínseca deles (Ryan \& Deci, 2016). Além disso, escolas que favorecem a motivação intrínseca podem beneficiar seus estudantes no processo de aprendizagem e estabelecimento de metas de aprendizagem (Froiland \& Worrel, 2016). Em um estudo qualitativo com estudantes de língua estrangeira, destacou-se que há uma influência positiva dos mentores em seus alunos, bem como foi evidenciada a importância da motivação intrínseca, emoções positivas, engajamento e perseverança no caminho para satisfação e sucesso no aprendizado (Oxford \& Bolaños-Sánchez, 2016).

O tipo de motivação de cada indivíduo relaciona-se com características suas, permanentes ou transitórias. A personalidade compõe um aspecto mais estável ao longo do tempo. Outros fatores podem indicar características mais transitórias que repercutem no tipo de motivação, sendo um exemplo, os construtos como o otimismo, esperança, entre outros (Luthans, Avolio, Avey, \& Norman, 2007). Em um estudo com universitários chineses obteve-se que a autodeterminação (consciência de si) explicou a identidade pessoal por meio da esperança no futuro (Zhou \& Kam, 2018). Outra pesquisa obteve que o capital psicológico (esperança, otimismo, autoeficácia e resiliência) no ambiente acadêmico é precursor-chave de desfechos acadêmicos adaptativos como, por exemplo, engajamento e sucesso acadêmico bem como motivação autêntica (Datu, King, \& Valdez, 2018).

Essas características individuais vão compor as propensões motivacionais que, juntamente com a forma como é percebida a satisfação de vida, auxilia a compreender o tipo de motivação demonstrado num determinado contexto. Por exemplo, características individuais positivas levam o sujeito a, quando diante de uma situação potencialmente adversa, ter a capacidade de identificar elementos positivos, evidenciando capacidade de lidar mais facilmente com situações estressantes (Affleck \& Tennen, 1996).

Com relação à personalidade dos indivíduos, contingências de autoestima são consideradas como traços intermediários contextualizados temporalmente. Sendo assim, quando o valor de si é atrelado a avaliações de outros, há uma maior inclinação ao neuroticismo, baixa autoestima, narcisismo ou uma combinação de todos esses aspectos. Evidências indicam que as diferentes características de personalidade dos estudantes implicam diferentes motivações e prioridades no âmbito acadêmico (Clark \& Schroth, 2010).

Existem vários modelos de avaliação da personalidade. Dentre estes, o Modelo dos Cinco Grandes Fatores (CGF) faz uma tentativa de buscar pontos em comum entre teorias e modelos e pode ser compreendido como uma versão moderna da Teoria dos Traços, que pressupõe que os indivíduos possuem predisposições comportamentais amplas frente a diferentes situações (Trentini, Hutz, \& Teixeira, 2009). Os cinco fatores são: Extroversão, Neuroticismo, Socialização, Realização e Abertura à Experiência. Foram encontradas evidências de que o fator Realização mostrou-se um bom preditor de sucesso acadêmico desde as séries iniciais até o ensino médio (Kyllonen, Lipnevich, Burrus, \& Roberts, 2014).

Características positivas da personalidade como, por exemplo, otimismo e esperança podem ser considerados importantes no ajuste psicológico diante de eventos estressores, sendo associados com menores desconfortos diante de situações adversas (Besser \& Zeigler-Hill, 
2014). Sendo assim, esses construtos, juntamente com a satisfação de vida, podem servir de indicativo acerca de como os indivíduos estão lidando com a realidade acadêmica e com a vida em geral.

O otimismo pode ser definido como uma disposição geral de esperar por desfechos positivos (Scheier \& Carver, 1985). Pessoas otimistas podem ter expectativas mais favoráveis do que pessimistas, e ser mais persistentes nos seus objetivos, obtendo melhores resultados nas suas ações (Tripathi \& Chaturvedi, 2014). Além disso, o otimismo mostrou-se um consistente preditor de manifestações emocionais de estresse em estudantes universitários (Fernández-González, González-Hernández, \& Trianes-Torres, 2017).

A esperança seria o pensamento dirigido a objetivos, sendo composta por rotas e agenciamentos (Lopez \& Snyder, 2003). Quando o indivíduo apresenta níveis maiores de esperança estabelece rotas mais sólidas para alcançar os objetivos desejados (Snyder, Shorey, Cheavens, Pulvers, \& Adams III, 2002).

A satisfação de vida, por sua vez, constitui-se por uma avaliação global de sentimentos e atitudes com relação à vida em um determinado ponto do tempo. Além disso, juntamente com afetos positivo e negativo, é indicador de bem-estar (Diener, 1984) e em estudantes universitários relaciona-se ao desempenho acadêmico (Pavot \& Diener, 2008). Foi demonstrado que em estudantes universitários maiores níveis de satisfação de vida associaram-se com envolvimento em projetos considerados prazerosos e com nível moderado de dificuldade (Palys \& Little, 1983), ou seja, atividades de acordo com a capacidade individual. Além disso, quando os estudantes sentem-se atendidos nas necessidades básicas de autonomia, relação e competência, existe um maior bem estar emocional e consequentemente maior satisfação de vida (Hagenauer, Gläser-Zikuda, \& Moschner, 2018).

Outros fatores externos também podem influenciar na motivação para a aprendizagem, um deles é o sexo. Existem evidências de que o sexo masculino tem mais dificuldade na organização de tarefas, planejamento e persistência, já o sexo feminino tende a apresentar comportamento mais ansioso (Edgar, 2015). Em um estudo que investigou a relação entre motivação e coping de estresse, a motivação indiretamente foi preditora da avaliação do estresse e de coping, assim as mulheres apresentaram maiores níveis de estresse, enquanto homens foram negativamente afetados utilizando estratégias de coping que não favoreciam o engajamento (Bonneville-Roussy, Evans, Verner-Filion, Vallerand, \& Bouffard, 2017).

Já com relação ao fator cultural, um estudo comparando amostras brasileiras e norte-americanas obteve que, enquanto os norte-americanos obtiveram maiores escores em esperança, satisfação de vida e afeto positivo, os brasileiros tiveram maiores escores em otimismo e afeto negativo, sendo apontadas possíveis diferenças culturais como explicação (Hutz, Midgett, Pacico, Bastianello, \& Zanon,
2014). A partir desse achado, reforça-se a importância de pesquisas com amostras brasileiras que avaliem estas variáveis, levando-se em consideração que a maior parte dos estudos relatados é de origem norte-americana ou europeia.

\section{Método}

\section{Participantes}

Participaram deste estudo 609 estudantes de universidades brasileiras públicas e privadas. A maior parte dos participantes era proveniente da região Sul (372), seguida pelas regiões Sudeste (132), Nordeste (43), CentroOeste (35) e Norte (27). Destes, 76,5\% (466) eram do sexo feminino, e a média de idade dos participantes de 22,8 anos $(D P=6,9)$. Além de estudar, 41,9\% (255) dos estudantes também trabalhavam.

\section{Instrumentos}

Escala de Autoestima Contingente, foi utilizada a versão traduzida e adaptada da Contingencies of Self-Worth Scale (CSWS) (Crocker et al.,2003). Trata-se de uma medida de 35 itens que avalia a variabilidade da autoestima nos domínios em que está ancorada. A escala é composta de sete subescalas que acessam as seguintes contingências: Aparência (Appearance), Competência Acadêmica (Academic Competence), Competição (Competition), Aprovação de Outros (Others' Approval), Virtude (Virtue), Suporte Familiar (Family Support) e Amor de Deus (God's Love). O participante indica o grau em que o item está de acordo com seu sentimento de autoestima por meio de uma escala de sete pontos. Os alfas dos fatores variaram de 0,71 a 0,96.

Escala de Autoestima (Rosenberg, 1979), adaptada para o português brasileiro (Hutz \& Zanon, 2011; Hutz, Zanon, \& Vazquez, 2014). Essa é uma medida unidimensional de dez itens que se relacionam a um conjunto de sentimentos de autoestima e auto aceitação e que avaliam a autoestima global. $\mathrm{O}$ alfa foi de 0,90

Escala de Motivação Acadêmica - EMA (Guimarães, Bzuneck, \& Joly, 2010). A EMA caracteriza a autopercepção de estudantes universitários quanto à motivação para frequentar a universidade. É um instrumento de autorrelato, composto por 29 itens que se distribuem em seis fatores: Desmotivação (alfa de 0,73), Motivação Extrínseca por Regulação Introjetada (alfa de 0,75), Motivação Extrínseca por regulação externa de frequência às aulas (alfa de 0,73), Motivação Extrínseca por regulação externa por recompensas sociais (alfa de 0,64 ), Motivação Extrínseca por Regulação Identificada (alfa de $0,54)$ e Motivação Intrínseca(alfa de 0,81 ). O alfa para a escala total foi de 0,82 )

Marcadores para Avaliação de Personalidade no Modelo dos Cinco Grandes Fatores (Hutz et al., 1998). Trata-se de uma escala composta por 64 descritores de traços que representam cinco fatores distintos: Socialização, Extroversão, Realização, Neuroticismo e Abertura. Os alfas de Cronbach variaram de 0,88 a 0,78 
nos diferentes domínios.

Revised Life Orientation Test - LOT-R (Bastianello \& Pacico, 2014). O teste foi desenvolvido por Scheier \& Carver (1985) e (Scheier, Carver, \& Bridges, 1994). É composto por 10 itens, sendo três afirmativas sobre otimismo, três sobre pessimismo e quatro itens filtro de escore não computado. A versão adaptada para o português brasileiro apresentou boa consistência interna $(0,76)$.

The Hope Index (Pacico, Zanon, Bastianello, \& Hutz, 2011; Staats, 1989). O instrumento avalia a esperança cognitiva. Constituído por 16 itens que referem eventos específicos, dividindo-se igualmente em duas subescalas ("esperança autocentrada" e "esperança altruísta”). No processo de adaptação, foi utilizada uma metodologia similar à da autora, resultando na adição de cinco itens que fazem parte da subescala "esperança autocentrada". O alfa da escala de esperança autocentrada foi de 0,83 e na escala de esperança altruísta foi de 0,81 .

Escala de Satisfação de Vida, foi utilizada uma adaptação da Escala de Satisfação de Vida - ESV (Diener, Emmons, Larsen, \& Griffin, 1985) para a população brasileira (Hutz, Zanon, \& Bardagi, 2014). É composta por cinco itens que avaliam a satisfação dos indivíduos com relação a sua vida.

\section{Procedimentos}

O estudo foi aprovado pelo comitê de ética no Instituto de Psicologia da Universidade Federal do
Rio Grande do Sul - UFRGS pelo parecer de número 2.594.753. A coleta de dados foi realizada por meio de aplicação presencial de questionários nas salas de aula de universidades públicas e privadas de Porto Alegre (38\%) e on-line por meio da plataforma Survey Monkey (62\%). Os participantes on-line foram recrutados através de postagem em rede social (Facebook) e divulgação via $e$-mail. $\mathrm{O}$ tempo de preenchimento médio dos instrumentos foi de mais ou menos 20 minutos tanto no formato presencial quanto no on-line.

\section{Resultados}

Com o objetivo de verificar o quanto os domínios da Contingencies of Self-Worth Scale se associavam com os diferentes tipos de motivação acadêmica foram realizadas análises de correlação. Obtiveram-se correlações significativas positivas entre Motivação Extrínseca - Regulação Introjetada e os domínios de Competição $(0,36)$, Competência Acadêmica $(0,38)$ e Aparência (0,30); entre Motivação Extrínseca frequência às aulas e o domínio Aparência $(0,30)$; e entre Regulação Identificada e Competência Acadêmica (Tabela 1).

Com relação aos marcadores de personalidade houve associação significativa do fator Neuroticismo com o domínio Aprovação de Outros $(0,34)$ (Tabela 2).

Tabela 1

Associação entre a Contingent Self-Worth Scale e a Escala de Motivação Acadêmica por meio do coeficiente de correlação de Pearson

\begin{tabular}{|c|c|c|c|c|c|c|c|}
\hline Self-Worth Scale & Desmotivação & $\begin{array}{l}\text { Motivação } \\
\text { Extrínseca: } \\
\text { Regulação } \\
\text { Introjetada }\end{array}$ & $\begin{array}{l}\text { Regulação } \\
\text { Externa: } \\
\text { Frequência } \\
\text { às Aulas } \\
\end{array}$ & $\begin{array}{c}\text { Regulação } \\
\text { Externa: } \\
\text { Recompensas } \\
\text { Sociais } \\
\end{array}$ & $\begin{array}{l}\text { Motivação } \\
\text { Extrínseca: } \\
\text { Regulação } \\
\text { Identificada } \\
\end{array}$ & $\begin{array}{l}\text { Motivação } \\
\text { Intrínseca }\end{array}$ & Total \\
\hline Amor de Deus & 0,05 & 0,07 & 0,05 & $-0,0$ & $0,14^{* *}$ & $-0,04$ & 0,06 \\
\hline Aprovação de Outros & $0,24^{* *}$ & $0,28^{* *}$ & $0,24^{* *}$ & $0,21^{* *}$ & $0,21^{* *}$ & $-0,19^{* *}$ & $0,31^{* *}$ \\
\hline Competição & $0,09^{*}$ & $0,35^{* *}$ & $0,19^{* *}$ & $0,14^{* *}$ & $0,24^{* *}$ & 0,01 & $0,32^{* *}$ \\
\hline $\begin{array}{l}\text { Competência } \\
\text { Acadêmica }\end{array}$ & 0,05 & $0,38^{* *}$ & $0,18^{* *}$ & $0,18^{* *}$ & $0,33^{* *}$ & 0,03 & $0,35^{* *}$ \\
\hline Aparência & $0,17^{* *}$ & $0,30^{* *}$ & $0,30^{* *}$ & $0,23^{* *}$ & $0,13^{* *}$ & $-0,14^{* *}$ & $0,31^{* *}$ \\
\hline Virtude & $-0,11^{* *}$ & 0,07 & $-0,01$ & $-0,11^{* *}$ & $0,20^{* *}$ & $0,16^{* *}$ & 0,05 \\
\hline Suporte Familiar & 0,01 & $0,21^{* *}$ & $0,11^{* *}$ & 0,05 & $0,19^{* *}$ & 0,06 & $0,20^{* *}$ \\
\hline
\end{tabular}

Nota. ${ }^{*} p<0,05 ;{ }^{* *} p<0,01$

As mulheres apresentaram escores significativamente mais elevados nos domínios Amor de Deus, Aprovação de Outros, Competência Acadêmica, Aparência e Suporte Familiar (Tabela 3).

A idade se associou significativamente com praticamente todos os domínios da escala de autoestima contingente, exceto Suporte Familiar. Com relação ao semestre do curso, somente o domínio Aparência foi significativo e negativo, indicando que quanto mais tempo no curso, menor a pontuação neste domínio (Tabela 4).

Os estudantes universitários que trabalham apresentam significativamente maior pontuação no domínio Amor de Deus e significativamente menor pontuação nos domínios Aprovação de Outros, Competência Acadêmica e Aparência (Tabela 5). 
Tabela 2

Associação entre a Contingent Self-Worth Scale e a Escala de Marcadores de Personalidade por meio do Coeficiente de Correlação de Pearson

\begin{tabular}{lccccc}
\hline \multicolumn{1}{c}{ Self-Worth Scale } & Socialização & Extroversão & Realização & Neuroticismo & Abertura \\
\hline Amor de Deus & $-0,07$ & $-0,01$ & $-0,05$ & $-0,21^{* *}$ & $-0,20^{* *}$ \\
Aprovação de Outros & $-0,13^{* *}$ & $-0,18^{* *}$ & $-0,27^{* *}$ & $0,34^{* *}$ & $-0,24^{* *}$ \\
Competição & $-0,13^{* *}$ & $-0,07$ & $-0,04$ & $0,16^{* *}$ & $-0,09^{*}$ \\
Competência Acadêmica & $-0,16^{* *}$ & $-0,04$ & $-0,10^{*}$ & $0,14^{* *}$ & $-0,18^{* *}$ \\
Aparência & $-0,06$ & $-0,10^{*}$ & $-0,18^{* *}$ & $0,29^{* *}$ & $-0,13^{* *}$ \\
Virtude & 0,02 & 0,01 & $0,15^{* *}$ & $-0,03$ & $-0,04$ \\
Suporte Familiar & $0,12^{* *}$ & $-0,08$ & 0,05 & $-0,01$ & $-0,05$ \\
\hline
\end{tabular}

Nota. ${ }^{*} p<0,05 ;{ }^{* *} p<0,01$

Tabela 3

Comparação da Contingent Self-Worth Scale entre os gêneros

\begin{tabular}{lcccc}
\hline \multirow{2}{*}{ Contingent Self-Worth Scale } & Masculino & & Feminino \\
\cline { 2 - 3 } Amor de Deus & Média \pm DP & Média \pm DP & $3.71 \pm 2.07$ & 0.01 \\
Aprovação de Outros & $2.61 \pm 1.89$ & $3.17 \pm 1.49$ & 0.01 \\
Competição & $3.62 \pm 1.47$ & $4.36 \pm 1.44$ & 0.58 \\
Competência Acadêmica & $4.29 \pm 1.34$ & $5.82 \pm 0.95$ & 0.01 \\
Aparência & $5.35 \pm 1.08$ & $5.03 \pm 1.40$ & 0.01 \\
Virtude & $4.42 \pm 1.40$ & $5.18 \pm 0.99$ & 0.51 \\
Suporte Familiar & $5.12 \pm 1.07$ & $5.31 \pm 0.99$ & 0.01 \\
\hline
\end{tabular}

Tabela 4

Associação da Contingent Self-Worth Scale com idade

\begin{tabular}{lcc}
\hline \multicolumn{1}{c}{ Contingent Self-Worth Scale } & Idade & \multicolumn{2}{c}{ Semestre } \\
\cline { 2 - 3 } Amor de Deus & $\mathrm{r}$ & $\mathrm{r}$ \\
Aprovação de Outros & $0.08(p=0.04)$ & $0.01(p=0.75)$ \\
Competição & $-0.14(p<0.01)$ & $-0.06(p=0.15)$ \\
Competência Acadêmica & $-0.08(p=0.03)$ & $-0.03(p=0.40)$ \\
Aparência & $-0.23(p<0.01)$ & $-0.05(p=0.22)$ \\
Virtude & $-0.28(p<0.01)$ & $-0.10(p=0.02)$ \\
Suporte Familiar & $0.10(p=0.09)$ & $0.08(p=0.05)$ \\
\hline
\end{tabular}

Nota. $r=$ coeficiente de correlação de Pearson

Tabela 5

Avaliação da Contingent Self-Worth Scale e Trabalho

\begin{tabular}{lcccc}
\hline \multirow{2}{*}{ Contingent Self-Worth Scale } & Trabalha & & Não Trabalha \\
\cline { 2 - 3 } Amor de Deus & Média \pm DP & Média \pm DP & 0,04 \\
Aprovação de Outros & $3,64 \pm 2,03$ & $3,29 \pm 2,09$ & 0,05 \\
Competição & $3,90 \pm 1,46$ & $4,15 \pm 1,52$ & 0,22 \\
Competência Acadêmica & $4,26 \pm 1,39$ & $4,40 \pm 1,43$ & 0,01 \\
Aparência & $5,52 \pm 1,01$ & $5,84 \pm 0,96$ & 0,05 \\
Virtude & $4,70 \pm 1,45$ & $5,03 \pm 1,39$ & 0,13 \\
Suporte Familiar & $5,24 \pm 0,98$ & $5,11 \pm 1,03$ & 0,73 \\
\hline
\end{tabular}


Para examinar a relação entre as diferentes contingências de autoestima e os outros construtos analisados, o Modelo de Regressão Linear Multivariado foi aplicado. Após o ajuste, permaneceram associadas significativamente com o domínio Amor de Deus as seguintes variáveis: otimismo, sexo, satisfação de vida, o domínio Motivação Extrínseca (Regulação Identificada) da Escala de Motivação Acadêmica e os marcadores de personalidade Neuroticismo e Abertura. Esse modelo explicou 16,8\% da variação do escore de Amor de Deus, sendo que as duas principais variáveis para essa explicação foram as Escalas de Otimismo e Satisfação de Vida.

Com o domínio Aprovação de Outros, permaneceram associadas após o ajuste as seguintes variáveis: Escalas de Otimismo e Rosenberg, o desempenho com base nas notas, os domínios Motivação Extrínseca (Regulação Identificada) e Motivação Extrínseca (Regulação Introjetada) da Escala de Motivação Acadêmica e os marcadores de personalidade Socialização, Realização e Extroversão. Este modelo explicou 35,9\% da variação do escore de Aprovação de Outros, sendo que as duas principais variáveis para essa explicação foram as Escalas de Rosenberg e a de Personalidade de Socialização.

Para o domínio Competição, permaneceram associados os domínios Motivação Extrínseca (Regulação Identificada) e Motivação Extrínseca (Regulação Introjetada) da Escala de Motivação Acadêmica e o marcador Neuroticismo de personalidade. Essas variáveis explicaram $17,1 \%$ do desfecho e a que mais contribui foi a Motivação Extrínseca por Regulação Introjetada.

Quanto ao domínio Competência Acadêmica as variáveis que permaneceram significativamente associadas foram: os domínios Motivação Extrínseca (Regulação Identificada) e Motivação Extrínseca (Regulação Introjetada) da Escala de Motivação Acadêmica, idade, sexo, trabalho, os marcadores de personalidade Abertura e Realização e desempenho com base nas notas. Esse modelo explicou $28,4 \%$ da variação do domínio Competência Acadêmica, com maior contribuição das variáveis Motivação Extrínseca por Regulação Introjetada da Escala de Motivação Acadêmica e marcador de personalidade Realização.

Para o domínio Aparência, ficaram significativamente associadas às seguintes variáveis: idade, sexo, os domínios Desmotivação, Motivação Extrínseca (Regulação Introjetada), Motivação Extrínseca (Regulação Externa Frequência às Aulas) da Escala de Motivação Acadêmica e percentil da Escala de Rosenberg. Essas variáveis explicam $25,5 \%$ do desfecho e as com maior contribuição foram a Escala de Rosenberg, Motivação Extrínseca por Regulação Introjetada e Motivação Extrínseca por Regulação Externa - Frequência às Aulas da Escala de Motivação Acadêmica.

Para Virtude, permaneceram associados após o ajuste os domínios Motivação Extrínseca (Regulação Identificada) e Motivação Extrínseca (Regulação
Externa - Recompensas Sociais) da Escala de Motivação Acadêmica e marcador de personalidade Realização. Essas variáveis explicaram 10\% do desfecho, com maior contribuição a Motivação Extrínseca por Regulação Identificada.

Por fim, as variáveis que permaneceram associadas com Suporte Familiar foram: sexo, Motivação Extrínseca (Regulação Introjetada) da Escala de Motivação Acadêmica e o marcador de personalidade Socialização. Essas variáveis explicaram $11 \%$ do escore de Suporte Familiar, com maior contribuição do sexo.

\section{Discussão}

Os resultados mostram que os domínios de contingência de autoestima se relacionaram mais significativamente com diferentes tipos de motivação extrínseca. Domínios de contingência considerados mais externos (Competição, Aparência e Competência Acadêmica) obtiveram correlação significativa, ainda que fraca, com motivação introjetada. Evidencia-se a tendência nessa amostra de que, quando diante de estímulo ou pressão interna por meio da culpa ou sentimentos similares, haverá maior necessidade de se atingir padrões mais elevados de competência, maior competitividade ou maior preocupação com a aparência. Em indivíduos que agem conforme sentimento de culpa fomentado por fatores externos as ações são controladas por padrões individuais e autoestima contingente externa (Deci \& Ryan, 1995). Um fator a ser considerado é que estudantes que apoiam a autoestima em contingência acadêmica estão muito mais sensíveis ao desempenho acadêmico considerado abaixo do desejável, podendo vivenciar sentimentos de desvalia e apresentar baixas na autoestima como resultado (Crocker, Karpinski, Quinn, \& Chase, 2003).

A correlação entre Motivação Extrínseca (Frequência às Aulas) e o domínio de contingência Aparência pode indicar provável dependência de um constante feedback externo por parte de pares e professores como forma de garantir a autoestima. Este achado está de acordo com a literatura, que indica que estudantes com questões como baixa autoestima, baixa autoestima corporal e baixa inteligência emocional estão mais suscetíveis a problemas como ansiedade (Abdollahi \& Talib, 2014)

Já a relação entre Regulação Identificada e Competência Acadêmica indica que a motivação, apesar de ainda externa, já está mais de acordo com os desejos. Nessa amostra, evidencia-se que quanto maior for a motivação por identificação, maior será a contingência no domínio Competência Acadêmica. Por se tratar de um ambiente universitário, pode-se pensar na importância de figuras que sirvam de modelo, levando aos alunos a adoção de padrões de comportamento com base na admiração por essas figuras.

Houve ainda correlação positiva fraca entre o fator de personalidade Neuroticismo e o domínio Aprovação 
de Outros; logo, nessa amostra, quanto maior a dependência de que haja aprovação externa, maiores os índices de Neuroticismo. Esse dado demonstra a relação, já amplamente ilustrada em pesquisas com estudantes universitários, desse fator com desfechos negativos como, por exemplo, baixa resolução de problemas sociais e sintomas depressivos (Koruklu, 2015; Song et al., 2018). Além disso, evidencia a necessidade de considerar aspectos internos dos estudantes com o intuito de fortalecê-los para lidar com questões e adversidades inerentes à vida acadêmica.

As mulheres nessa amostra demonstraram estarem mais vulneráveis na maioria das vezes a domínios de contingência ditos externos (Aprovação de Outros, Aparência, Competência Acadêmica e Suporte Familiar), indicando maior vulnerabilidade a fatores externos. Este achado está de acordo com o relato de estudos prévios (Crocker et al., 2003; García-Soriano, Belloch, \& Castañeiras, 2010). Estudos apontam que as mulheres estão mais propensas a basear seu valor com base na aparência como uma característica que as define e diferencia em diferentes aspectos como econômico e social (Judge, Hurst, \& Simon, 2009) e até mesmo com relação à saúde física (Langlois et al., 2000; Noser \& Zeigler-Hill, 2014). Além disso, evidências apontam que mulheres com motivações de ordem externa estão mais propensas a pressões culturais para serem magras e a distúrbios alimentares do que mulheres cuja motivação é mais autônoma (intrínseca) (Pelletier, Dion, \& Lévesque, 2004). No que tange à competência acadêmica, vários estudos apontam que o sexo feminino tende a se sair melhor no contexto acadêmico, entretanto são muitas vezes mais vulneráveis a questões internas que produzem desconforto (Langlois et al., 2000).

Com relação à idade, houve associação significativa negativa com todos os domínios (exceto Amor de Deus e Virtude). Apesar das correlações terem sido fracas, observa-se uma tendência de que quanto maior a idade menor a vulnerabilidade a domínios de contingência externa, o que não se mostrou em domínios considerados internos. Outro fator interessante observado nessa amostra é que os participantes que trabalham apresentaram menor pontuação nos domínios Aprovação de Outros, Competência Acadêmica e Aparência. Isso pode ser explicado em parte devido ao fato do estudo ser apenas uma parte da vida desses indivíduos, obtendo outras fontes de manutenção da autoestima para além do ambiente universitário.

As análises de regressão mostraram que os domínios que obtiveram maior poder de explicação no modelo foram Aprovação de Outros (35,9\%), Competência Acadêmica (28,4\%) e Aparência (25,5\%). Considerando o momento de vida que desses estudantes - saída da adolescência e entrada no mundo adulto -, destacaram-se domínios pertinentes nessa fase do desenvolvimento. Considerando-se que a autoestima obtém variações ao longo do desenvolvimento e que há uma queda em seus níveis na época da adolescência (Robins \& Trzesniewski, 2005), pode-se pensar que a maior parte dos estudantes participantes da amostra contava com idade na casa dos 20 anos, portanto ainda mais vulneráveis à avaliação e aprovação de outros. Além disso, trata-se de domínios mais externos de contingência que podem ser associados à motivação não autônoma e controlada, evidenciando sentimentos de sentir-se controlado, estresse e menor bem-estar (Deci \& Ryan, 2000). Outro fator a ser considerado em situações de contingência externa é que isso pode levar ao não alcance de objetivos e a menor performance acadêmica (Lee, 2005).

A associação mais evidente do domínio Aprovação de Outros com autoestima global e o marcador de personalidade Socialização indica domínios de contingência mais externos, fazendo sentido pensar que quanto maior a necessidade de aprovação dos outros, menor o índice de autoestima global, bem como maiores os níveis de Socialização como uma forma de responder às contingências externas. Um estudo longitudinal desde a adolescência até a idade adulta indicou que existem ligações de longo termo entre construtos sociais adultos, aprovação objetiva ou subjetiva de pares e autoestima; sendo assim, a forma como as relações se estabelecem desde a juventude acaba gerando impacto ao longo da vida adulta (Gruenenfelder-Steiger, Harris, \& Fend, 2016).

Por se tratar de uma amostra de estudantes universitários, era esperado que o domínio Competência Acadêmica fosse um dos domínios de destaque, já que se trata de um momento de vida decisivo e sensível na vida dos estudantes. As variáveis que mais contribuíram nesse modelo foram Motivação Extrínseca por Regulação Introjetada e marcador de personalidade Realização. O tipo de motivação, que engloba sentimento de culpa e similares, evidencia o quanto jovens que apoiam sua autoestima e valor nesse tipo de contingência estão sujeitos quando não atingem o padrão idealizado por si mesmo e por pessoas cujos laços são considerados importantes. Além disso, evidencia-se a característica de personalidade correlacionada com a organização e direção do comportamento diante do desempenho acadêmico (Hutz et al., 1998).

O domínio Aparência teve contribuição da Escala de Rosenberg, Motivação Extrínseca - Regulação Introjetada e Motivação Extrínseca - Frequência às Aulas, indicando que há uma associação negativa da contingência da aparência com a autoestima global, bem como a contribuição positiva de motivações de cunho externo que envolve sentimento de culpa. Esse tipo de associação já foi apontado como indicador que autoestima global tem uma relação forte e estável com o domínio da aparência ao longo do desenvolvimento (von Soest, 2015).

Os resultados dessa pesquisa indicam que na amostra estudada houve associação de domínios mais externos de contingência de autoestima com tipos de Motivação Extrínseca no âmbito acadêmico (Competência 
Acadêmica, Competição e Aparência). Esses domínios são prevalentes nesse meio, que conta com um maior número de estudantes jovens e muitas vezes vulneráveis a pressões externas. Esse dado é importante e faz pensar na necessidade de intervenções em nível educacional que estimulem os estudantes à adoção de motivações mais intrínsecas para aprendizagem. Estudos apontam que isso também pode ser considerado um papel de quem ensina, no sentido de que a Motivação Intrínseca pode ser facilitada através do comportamento dos professores (Ryan \& Deci, 2000). Quando há estímulo intelectual por parte dos professores, os alunos podem incrementar seu senso de autonomia e com isso se cria um ambiente mais favorável ao aprendizado (Bolkan, 2015; Broeck, Vansteenkiste, Witte, Soenens, \& Lens, 2010).

\section{Considerações Finais}

Este estudo oferece algumas limitações que são o elevado número de estudantes participantes provenientes da região sul, bem como o fato de a maior parte da amostra ser composta por mulheres. Apesar de ser sabido que, de acordo com o Censo Educacional de 2015 (Ministério da Educação - INEP), há uma predominância de mulheres cursando o ensino superior e um maior número de mulheres que finalizam os estudos, seria interessante investigar essas variáveis em uma amostra mais representativa da população. Também seria interessante fazer comparações entre regiões do país e entre estudantes de universidades públicas e privadas.

\section{Referências}

Abdollahi, A., \& Talib, M. A. (2014). Sedentary behaviour and social anxiety in obese individuals: The mediating role of body esteem. Psychology, Health \& Medicine, 20, 205-209. doi: 10.1080/13548506.2014.913799

Affleck, G., \& Tennen, H. (1996). Construing benefits from adversity: Adaptotional significance and disposltional underpinnings. Journal of Personality, 64(4), 899-922. doi: 10.1111/j.1467-6494.1996.tb00948.x

Bastianello, M., \& Pacico, J. C. (2014). Otimismo. Em Avaliação em Psicologia Positiva (p. 95-100). Artmed.

Besser, A., \& Zeigler-Hill, V. (2014). Positive personality features and stress among first-year university students: Implicatons for psycholoical distress, functional impairment, and self-esteem. Self and Identity, 13(1), 24-44. doi: 10.1080/15298868.2012.736690

Bolkan, S. (2015). Intellectually stimulating students' intrinsicmotivation: The mediating influence of affectivelearning and student engagement. Communication Reports, 28(2), 89-91. doi: 10.1080/08934215.2014.962752

Bonneville-Roussy, A., Evans, P., Verner-Filion, J., Vallerand, R. J., \& Bouffard, T. (2017). Motivation and coping with the stress of assessment: Gender differences in outcomes for university students. Contemporary Educational Psychology, 48, 28-42. doi: 10.1016/j. cedpsych.2016.08.003

Broeck, A., Vansteenkiste, M., Witte, H., Soenens, B., \& Lens, W. (2010). Capturing autonomy, competence, and relatedness at work: Construction and initial validation of the Work-related Basic Need Satisfaction scale. Journal of Occupational and Organizational Psychology, 83(4), 981-1002. doi: 10.1348/096317909X481382

Clark, M. H., \& Schroth, C. A. (2010). Examining relationships between academic motivation and personality among college students. Learning and Individual Differences, 20(1), 19-24.

Crocker, J., Karpinski, A., Quinn, D. M., \& Chase, S. K. (2003). When grades determine self-worth: Consequences of contingent self-worth for male and female engineering and psychology majors. Journal of Personality and Social Psychology, 85(3), 507-516. doi: 10.1037/00223514.85.3.507

Crocker, J., Luhtanen, R. K., Cooper, M. L., \& Bouvrette, A. (2003). Contingencies of self-worth in college students: Theory and measurement. Journal of Personality and Social Psychology, 85(5), 894-908. doi: 10.1037\%2F0022-3514.85.5.894

Crocker, J., \& Park, L. E. (2004). The costly pursuit of self-esteem. Psychological Bulletin, 130(3), 392-414. doi: 10.1037/00332909.130.3.392

Crocker, J., \& Wolfe, C. T. (2001). Contingencies of self-worth. Psychological Review, 108(3), 593-623. doi: 10.1037/0033-295X.108.3.593

Cunha, S. M., \& Carrilho, D. M. (2005). O processo de adaptação ao ensino superior e o rendimento acadêmico. Psicologia Escolar e Educacional, 9(2), 215-224. doi: 10.1590/S1413-85572005000200004

Datu, J. A. D., King, R. B., \& Valdez, J. P. M. (2018). Psychological capital bolsters motivation, engagement, and achievement: Crosssectional and longitudinal studies. The Journal of Positive Psychology, 13(3), 260-270. doi: 10.1080/17439760.2016.1257056

Deci, E. L., \& Ryan, R. M. (1995). Efficacy, Agency, and Self-Esteem (1995 edition; M. H. Kernis, Org.). New York: Springer.

Diener, E., Emmons, R. A., Larsen, R. J., \& Griffin, S. (1985). The satisfaction wih life scale. Journal of Personality Assessment, 49, 71-75. doi: 10.1207/s15327752jpa4901_13

Diener, Ed. (1984). Subjective Wēll-Being. Psychological Bulletin, 95(3), 542-575. Recuperado de http://labs.psychology.illinois.edu/ ediener/ Documents/Diener_1984.pdf

Edgar, S. (2015). Identifying the influence of gender on motivation and engagement levels in student physiotherapists. Medical Teacher, 37(4), 348-353. doi: 10.3109/0142159X.2014.948829

Fernández-González, L., González-Hernández, A., \& Trianes-Torres, M. V. (2017). Relaciones entre estrés académico, apoyo social, optimismo-pesimismo y autoestima en estudiantes universitarios. Electronic Journal of Research in Education Psychology, 13(35). doi: 10.14204/ejrep.35.14053

Froiland, J. M., \& Worrel, F. C. (2016). Intrinsic motivation, learning goals, engagement, and achievement in a diverse high school. Psychology in the Schools, 53(3), 321-336. doi: 10.1002/pits.21901 
García-Soriano, G., Belloch, A., \& Castañeiras, C. (2010). Áreas en las que basamos nuestra autoestima y su influencia sobre el bienestar emocional: Validación de la escala de contingencias de la autoestima para su uso en castellano. Revista Argentina de Clínica Psicológica, XIX(1), 5-13. Recuperado de https://www.redalyc.org/pdf/2819/281921797001.pdf

Gilman, R., \& Anderman, E. M. (2006). The relationship between relative levels of motivation and intrapersonal, interpersonal, and academic functioning among older adolescents. Journal of School Psychology, 44(5), 375-391. doi: 10.1016/j.jsp.2006.03.004

Gruenenfelder-Steiger, A. E., Harris, M. A., \& Fend, H. A. (20161003). Subjective and objective peer approval evaluations and self-esteem development: A test of reciprocal, prospective, and long-term effects. Developmental Psychology, 52(10), 1563. doi: 10.1037/dev0000147

Guimarães, S., Bzuneck, J., \& Joly, M. (2010). Escala de Avaliação da Motivação para Universidade (AMU). Manuscrito não Publicado. Universidade Estadual de Londrina/Universidade São Francisco.

Hagenauer, G., Gläser-Zikuda, M., \& Moschner, B. (2018). University students' emotions, life-satisfaction and study commitment: A selfdetermination theoretical perspective. Journal of Further and Higher Education, 42(6), 808-826. doi: 10.1080/0309877X.2017.1323189

Hutz, C. S., Midgett, A., Pacico, J. C., Bastianello, M. R., \& Zanon, C. (2014). The relationship of hope, optimism, self-esteem, subjective well-being, and personality in Brazilians and Americans. Psychology, 05(06), 514-522. doi: 10.4236/psych.2014.56061

Hutz, C. S. \& Zanon, C. (2011). Revisão da apadtação, validação e normatização da escala de autoestima de Rosenberg: Revision of the adaptation, validation, and normatization of the Roserberg self-esteem scale. Avaliação Psicológica, 10(1), 41-49.

Hutz, C. S., Nunes, C. H., Silveira, A. D., Serra, J., Anton, M., \& Wieczorek, L. S. (1998). O desenvolvimento de marcadores para a avaliação da personalidade no modelo dos cinco grandes fatores. Psicologia: Reflexão e Crítica, 11(2), 395-411. Recuperado de https:// www.redalyc.org/pdf/188/18811215.pdf

Hutz, C. S., Zanon, C., \& Bardagi, M. P. (2014). Satisfação de vida. Em Avaliação em Psicologia Positiva (1o ed, p. 43-48). Porto Alegre: Artmed.

Hutz, C. S., Zanon, C., \& Vazquez, A. C. S. (2014). Escala de Autoestima de Rosenberg. In Avaliação em psicologia Positiva. Porto Alegre: Artmed.

Judge, T. A., Hurst, C., \& Simon, L. S. (2009). Does it pay to be smart, attractive, or confident (or all three)? Relationships among general mental ability, physical attractiveness, core self-evaluations, and income. Journal of Applied Psychology, 94(3), 742-755. doi:10.1037/ a0015497

Koruklu, N. (2015). Personality and social problem solving: The mediating role of self-esteem. Educational Sciences: Theory and Practice, 15(2), 481-487. doi: 10.12738/estp.2015.2.2601

Kyllonen, P. C., Lipnevich, A. A., Burrus, J., \& Roberts, R. D. (2014). Personality, motivation, and college readiness: A prospectus for assessment and development: personality, motivation, and college readiness. ETS Research Report Series, 2014(1), 1-48. doi: 10.1002/ ets2.12004

Langlois, J. H., Kalakanis, L., Rubenstein, A. J., Larson, A., Hallam, M., \& Smoot, M. (2000). Maxims or myths of beauty? A meta-analytic and theoretical review. Psychological Bulletin, 126(3), 390-423. doi: 10.1037/0033-2909.126.3.390 ER

Lee, E. (2005). The relationship of motivation and flow experience to academic procrastination in university students. The Journal of Genetic Psychology, 166(1), 5-14. doi: 10.3200/GNTP.166.1.5-15

Lepper, M. R., \& Henderlong, J. (2000). Turning "play" into "work" and "work" into "play": 25 years of research on intrinsic versus extrinsic motivation. In Intrinsic and extrinsic motivation: The search for optimal motivation and performance (p. 257-307). San Diego CA: Academic Press.

Lopez, F. G., Thorne, B., Schoenecker, S., Siffert, K., Chaliman, R., \& Castleberry, E. (2014). Externally contingent and unstable self-worth as predictors of depression in college women: A 3-Month Study. Journal of College Counseling, 17(2), 102-115. doi: 10.1002/j.21611882.2014.00051.x

Lopez, S. J., \& Snyder, C. R. (2003). Positive Psychological Assessment: A Handbook of Models and Measures. American Psychological Association.

Luthans, F., Avolio, B. J., Avey, J. B., \& Norman, S. M. (2007). Positive psychological capital: Measurement and relationship with performance and satisfaction. Personnel Psychology, 60, 541-572. doi: 10.1111/j.1744-6570.2007.00083.x

Maricutoiu, L. P., Macsinga, I., Rusu, S., VÎrga, D., \& Sava, F. A. (2012). Adaptation and validation of the contingencies of self-worth scale on a Romanian student sample. Cognition, Brain, Behavior, 16(1), 121.

McDermott, P. A., Mordell, M., \& Stoltzfus, J. C. (2001). The organization of student performance in American schools: Discipline, motivation, verbal learning, nonverbal learning. Journal of Educational Psychology, 93(1), 65-76. doi: 10.1037/0022-0663.93.1.65

Noser, A., \& Zeigler-Hill, V. (2014). Investing in the ideal: Does objectified body consciousness mediate the association between appearance contingent self-worth and appearance self-esteem in women? Body Image, 11(2), 119-125. doi: 10.1016/j.bodyim.2013.11.006

Oxford, R. L., \& Bolaños-Sánchez, D. (2016). A tale of two learners: Discovering mentoring, motivation, emotions, engagement, and perseverance. Em C. Gkonou, D. Tatzl, \& S. Mercer (Eds.), New Directions in Language Learning Psychology (p. 113-134). doi: 10.1007/978-3-319-23491-5_8

Pacico, J. C., Zanon, C., Bastianello, M. R., \& Hutz, C. S. (2011). Adaptation and validation of The Hope Index for Brazilian adolescents. Psicologia: Reflexão e Crítica, 24(4), 666-670. doi: 10.1590/S0102-79722011000400006

Palys, T. S., \& Little, B. R. (1983). Perceived life satisfaction and the organization of personal project systems. Journal of Personality and Social Psychology, 44(6), 1221-1230. doi: 10.1037/0022-3514.44.6.1221

Pavot, W., \& Diener, E. (2008). The Satisfaction With Life Scale and the emerging construct of life satisfaction. The Journal of Positive Psychology, 3(2), 137-152. doi: 10.1080/17439760701756946

Pelletier, L. G., Dion, S., \& Lévesque, C. (2004). Can self-determination help protect women against sociocultural influences about body image and reduce their risk of experiencing bulimic symptoms? Journal of Social and Clinical Psychology, 23(1), 61-88. doi: 10.1521/ jscp.23.1.61.26990

Robins, R. W., \& Trzesniewski, K. H. (2005). Self-esteem development across the lifespan. Current Directions in Psychological Science, 14, 158162. doi: 10.1111/j.0963-7214.2005.00353.x

Rosenberg, M. (1979). Society and the adolescent self-image, Rev. Ed. Middletown, CT, England: Wesleyan University Press.

Ryan, R. M., \& Deci, E. L. (2000). Self-determination theory and the facilitation of intrinsic motivation, social development, and well-being. The American Psychologist, 55(1), 68-78. doi: 10.1037/0003-066X.55.1.68

Ryan, R. M., \& Deci, E. L. (2016). Facilitating and hindering motivation, learning, and well-being in schools-research and observations from self-determination theory. In Handbook of Motivation at School (p. 96-119). New York: Routledge. 
Ryan, R. M., Deci, E. L., \& Grolnick, W. S. (1995). Autonomy, relatedness, and the self: Their relation to development and psychopathology. Em D. Cicchetti \& D. J. Cohen (Orgs.), Developmental psychopathology, Vol. 1: Theory and methods (p. 618-655). Oxford, England: John Wiley \& Sons.

Sanchez, D. T., \& Crocker, J. (2005). How investment in gender ideals affects well-being: The role of external contingencies of self-worth. Psychology of Women Quarterly, 29(1), 63-77. doi: 10.1111/j.1471-6402.2005.00169.x

Scheier, M. F., \& Carver, C. S. (1985). Optimism, coping, and health: Assessment and implications of generalized outcome expectancies. Health Psychology: Official Journal of the Division of Health Psychology, American Psychological Association, 4(3), 219-247.

Scheier, M. F., Carver, C. S., \& Bridges, M. W. (1994). Distinguishing optimism from neuroticism (and trait anxiety, self-mastery, and selfesteem): A reevaluation of the Life Orientation Test. Journal of Personality and Social Psychology, 67(6), 1063-1078. doi: 10.1037//00223514.67.6.1063

Sheldon, K. M., Ryan, R. M., Rawsthorne, L. J., \& Ilardi, B. (1997). Trait self and true self: Cross-role variation in the Big-Five personality traits and its relations with psychological authenticity and subjective well-being. Journal of Personality and Social Psychology, 73(6), 13801393. doi: 10.1037/0022-3514.73.6.1380

Snyder, C. R., Shorey, H. S., Cheavens, J., Pulvers, K. M., \& Adams III, V. H. (2002). Hope and academic sucess in college. Journal of Educational Psychology, 4, 820-826.

Song, Y., Tang, S., Guo, Q., Liu, Z., Chen, H., \& Huang, Y. (2018). Two-year prospective study of natural course and risk factors of depressive symptoms in college students. Chinese Mental Health Journal, 32(1), 64-71. doi: 10.3969/j.issn.1000-6729.2018.01.012

Staats, S. (1989). A comparison of two self-report measures for adults. Journal of Personality Assessment, 53(2), 366-375.doi:10.1207/ s15327752jpa5302_13

Trentini, C. M., Hutz, C. S., \& Teixeira, M. A. P. (2009). Correlações entre a EFN - Escala Fatorial de Neuroticismo e o IFP - Inventário Fatorial de Personalidade. Avaliação Psicológica, 8(2), 209-217.

Tripathi, A., \& Chaturvedi, K. R. (2014). Optimism: A factor intrinsic motivation and achievement. African International Journal of Research in Management, 2(4), 1-8. Recuperado de http://www.ifsmrc.org/?q=journals/management/volume2/issue4/Optimism

von Soest, T. (20150713). The development of global and domain-specific self-esteem from age 13 to 31. doi: 10.1037/pspp0000060

Zhou, M., \& Kam, C. C. S. (2018). Self-determination and personal identity in university students: The mediating role of future orientation. The Spanish Journal of Psychology, 21(14), 1-10. doi: 10.1017/sjp.2018.17

\section{Sobre os autores}

Daniela Bergesch D’Incao Marrone é psicóloga clínica, mestre em Psicologia pela UFRGS e doutoranda no Instituto de psicologia da UFRGS.

Claudio Simon Hutz é psicólogo, com doutorado na University of Iowa (USA), pós-doutorado na Arizona State University (USA), professor titular na UFRGS e coordenador do Laboratório de Mensuração do Instituto de Psicologia (UFRGS). 